\title{
Modulation of upper airway collapsibility during sleep: influence of respiratory phase and flow regimen
}

\author{
HARTMUT SCHNEIDER, ${ }^{1}$ AN BOUDEWYNS, ${ }^{2}$ PHILIP L. SMITH,${ }^{1}$ \\ CHRISTOPHER P. O'DONNELL, ${ }^{1}$ SEBASTIAN CANISIUS, ${ }^{3}$ AXEL STAMMNITZ, ${ }^{3}$ \\ LAWRENCE ALLAN, ${ }^{1}$ AND ALAN R. SCHWARTZ ${ }^{1}$ \\ ${ }^{3}$ Sleep Laboratory, Phillips-University of Marburg, 35032 Marburg, Germany; ${ }^{2}$ Department of \\ Otorhinolaryngology, Head and Neck Surgery, University Hospital Antwerp, B-2650 Edegem, \\ Belgium; and ${ }^{1}$ Johns Hopkins Sleep Disorders Center, Division of Pulmonary and Critical Care \\ Medicine, Johns Hopkins University School of Medicine, Baltimore, Maryland 21224
}

Received 12 September 2001; accepted in final form 24 June 2002

\begin{abstract}
Schneider, Hartmut, An Boudewyns, Philip L. Smith, Christopher P. O'Donnell, Sebastian Canisius, Axel Stammnitz, Lawrence Allan, and Alan R. Schwartz. Modulation of upper airway collapsibility during sleep: influence of respiratory phase and flow regimen. $J$ Appl Physiol 93: 1365-1376, 2002. First published July 5, 2002; 10.1152/ japplphysiol.00942.2001.-We hypothesized that upper airway collapsibility is modulated dynamically throughout the respiratory cycle in sleeping humans by alterations in respiratory phase and/or airflow regimen. To test this hypothesis, critical pressures were derived from upper airway pressureflow relationships in six tracheostomized patients with obstructive sleep apnea. Pressure-flow relationships were generated by varying the pressure at the trachea and nose during tracheostomy (inspiration and expiration) (comparison $A$ ) and nasal (inspiration only) breathing (comparison $B$ ), respectively. When a constant airflow regimen was maintained throughout the respiratory cycle (tracheostomy breathing), a small yet significant decrease in critical pressure was found at the inspiratory vs. end- and peak-expiratory time point $[7.1 \pm 1.6(\mathrm{SE})$ to $6.6 \pm$ 1.9 to $6.1 \pm 1.9 \mathrm{cmH}_{2} \mathrm{O}$, respectively; $P<0.05$ ], indicating that phasic factors exerted only a modest influence on upper airway collapsibility. In contrast, we found that the inspiratory critical pressure fell markedly during nasal vs. tracheostomy breathing $\left[1.1 \pm 1.5(\mathrm{SE})\right.$ vs. $\left.6.1 \pm 1.9 \mathrm{cmH}_{2} \mathrm{O} ; P<0.01\right]$, indicating that upper airway collapsibility is markedly influenced by differences in airflow regimen. Tracheostomy breathing was also associated with a reduction in both phasic and tonic genioglossal muscle activity during sleep. Our findings indicate that both phasic factors and airflow regimen modulate upper airway collapsibility dynamically and suggest that neuromuscular responses to alterations in airflow regimen can markedly lower upper airway collapsibility during inspiration.
\end{abstract}

obstructive sleep apnea; critical pressure; collapsibility; tracheostomy

IN OBSTRUCTIVE SLEEP apnea, the upper airway collapses and obstructs airflow periodically during sleep $(33,49)$. Although the mechanism for obstruction is not well

Address for reprint requests and other correspondence: A. R. Schwartz, Division of Pulmonary and Critical Care Medicine, The Johns Hopkins Asthma and Allergy Center, 5501 Hopkins Bayview Circle, Baltimore, Maryland 21224 (E-mail: aschwar2@jhmi.edu). understood, investigators initially focused on elucidating the mechanism for upper airway obstruction during inspiration (33). More recently, investigators found evidence in humans for upper airway obstruction during expiration $(3,21,22,38)$ and demonstrated in animals that the degree of obstruction increases during this phase of respiration (45). Nevertheless, the modulation of upper airway function has not been systematically examined in sleeping individuals throughout the respiratory cycle.

Previously, investigators have assessed upper airway function during sleep by measuring the critical closing pressure (Pcrit), an index of pharyngeal collapsibility $(6,9,10,27,40,44,49)$. These measurements were derived from an analysis of pressure-flow relationships obtained during inspiration when phasic increases in neuromuscular activity are known to occur (33). Such phasic activity can be further augmented by reflex neuromuscular responses to negative pressure, airway occlusion, and alterations in gas exchange (1, 11, 33, 50, 58). Moreover, increased neuromuscular activity has been associated with reductions in upper airway Pcrit values in animals $(35,45,46)$. Thus it is likely that previous measurements of Pcrit during inspiration in sleeping humans reflected the influence of phasic neuromuscular activity and reflex mechanisms controlling upper airway collapsibility.

In contrast, both neuromuscular and mechanical differences may alter upper airway collapsibility during expiration. The Pcrit might increase as neuromuscular activity falls to tonic levels during this phase of respiration (45). Under conditions of reduced neuromuscular activity, the Pcrit might be further influenced by mechanical factors, including a reversal in airflow direction and declining lung volume $(52,54)$ throughout the expiratory phase. It is, therefore, likely that both neuromuscular and mechanical factors alter upper airway Pcrit values during expiration, although such

The costs of publication of this article were defrayed in part by the payment of page charges. The article must therefore be hereby marked "advertisement" in accordance with 18 U.S.C. Section 1734 solely to indicate this fact. 

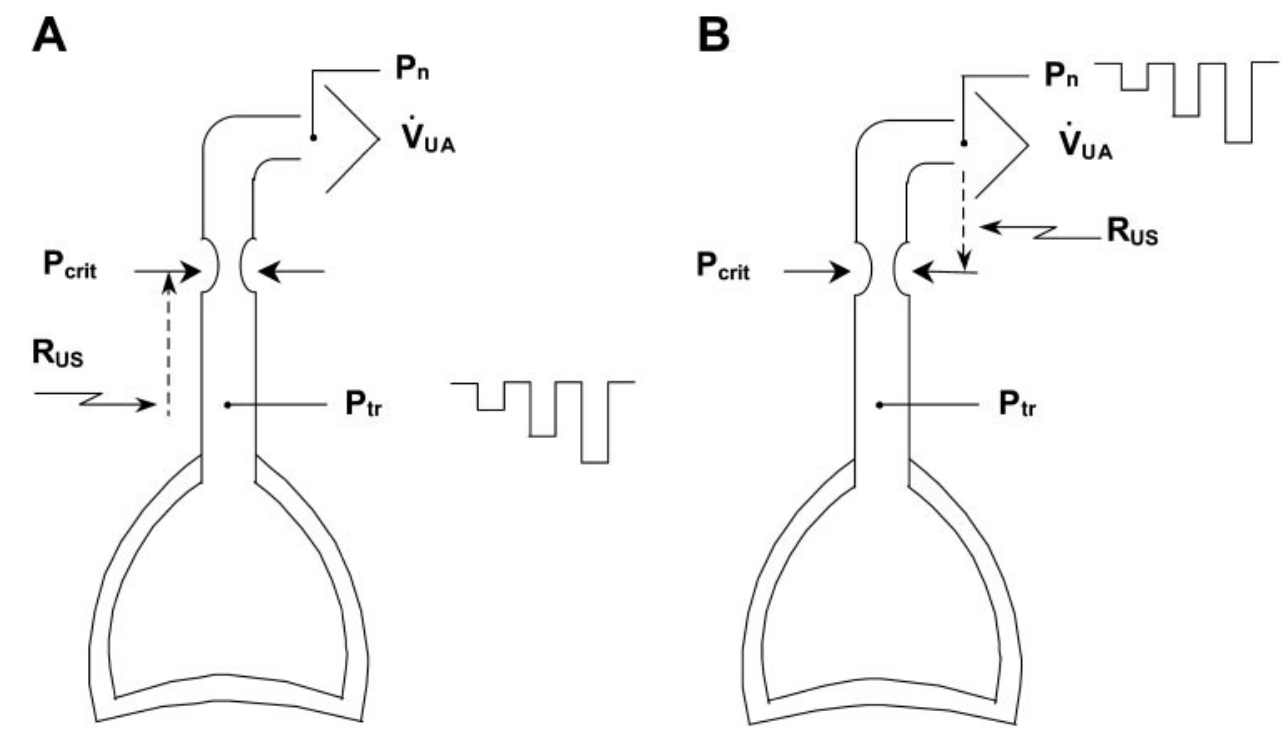

Fig. 1. The experimental setup and protocols are illustrated for conditions in which the upstream pressure (Pus) is systematically varied at the trachea $(\Delta \mathrm{Ptr} ; A)$ and at the nose $(\Delta \mathrm{Pn} ; B)$. In each case, airflow through the upper airway (Vua) is measured at various levels of Pus. Pressure-flow relationships are analyzed to calculate the critical pressure (Pcrit) and upstream resistance (Rus), as detailed in the text. Pcrit corresponds to the site of upper airway collapse. Rus corresponds to the nasal segment when $\mathrm{Pn}$ is varied and to the tracheal segment when Ptr is varied (see dashed vertical arrows). measurements have not yet been reported in sleeping humans.

The present study was designed to examine those factors modulating upper airway collapsibility in sleeping humans throughout the respiratory cycle. Based on previous findings in the isolated upper airway of animals $(45,46)$, we hypothesized that upper airway collapsibility is modulated dynamically throughout the respiratory cycle in sleeping humans by alterations in either respiratory phase or airflow regimen. To examine the effect of phasic factors, Pcrit values were derived from tracheal pressure (Ptr)-flow relationships at specific phases of the respiratory cycle in the isolated upper airway segment of six tracheostomized patients with obstructive sleep apnea. The influence of airflow regimen on Pcrit values was then examined by reversing the direction of upper airway flow during inspiration. Our findings indicate that upper airway collapsibility is modulated dynamically throughout the respiratory cycle and that both phasic mechanisms and differences in airflow regimen play a role. Moreover, our methods now provide a new model for estimating the mechanical loads imposed by the upper airway on the respiratory system during each phase of respiration.

\section{Background}

In previous studies, the upper airway has been modeled as a simple, collapsible conduit (Starling resistor) during sleep $(10,43,49)$. As discussed below, this model provides a generalized approach for determining the Pcrit during inspiration and expiration, based on an analysis of pressure-flow relationships in the upper airway segment. A major feature of this model is that it describes the conditions leading to alterations in upper airway patency. Specifically, the model predicts that the airway would completely occlude whenever pressures both upstream (Pus) and downstream (Pds) fall below a Pcrit. Under these circumstances, no flow could pass through the airway as long as Pcrit $>$ Pus $\geq$ Pds.
As the Pus is raised above the Pcrit, however, the upper airway would no longer remain occluded. Rather, the Starling resistor model predicts that a flow-limited state would ensue as long as the Pds remains below Pcrit (Pus $>$ Pcrit $>$ Pds) $(29,30,32$, 57). Under conditions of flow limitation, investigators have also demonstrated that flow through the upper airway rises linearly with elevations in Pus, regardless of the Pds level $(10,43,44,49)$. Thus the Starling resistor model predicts that the airway will occlude when Pus and Pds remain below a Pcrit and that flow limitation will result in linear increases in airflow as the Pus is raised above the Pcrit. ${ }^{1}$

The Starling resistor model can be used to describe airflow dynamics in the upper airway throughout the respiratory cycle. During inspiration, the nasal pressure $(\mathrm{Pn})$ and Ptr are considered to be the pressures upstream and downstream to the upper airway, respectively (Fig. 1A). As mentioned, as long as the Ptr falls below the Pcrit during inspiration, the upstream Pn will determine whether the upper airway occludes (Pn $<$ Pcrit, Ptr) or limits flow (Pn $>$ Pcrit $>$ Ptr). During expiration, however, the Ptr now becomes the Pus, driving flow out the upper airway to atmospheric pressure downstream at the nose (Fig. $1 B$ ). Despite the reversal in airflow direction, analogous flow dynamics are predicted during expiration, depending on Pus, Pds, and Pcrit levels. Specifically, the upper airway will either limit flow or occlude whenever the Pcrit is positive (provided that the downstream Pn remains atmospheric). Under these circumstances, airflow will cease (the airway will occlude) whenever the upstream Ptr is below the Pcrit (Pcrit $>$ Ptr $>$ Pn) and will increase linearly as the Ptr is increased above the Pcrit $(\mathrm{Ptr}>\mathrm{Pcrit}>\mathrm{Pn}=0$ ). Thus upper airway flow dynamics during tracheostomy breathing are predicted to

\footnotetext{
${ }^{1}$ The conditions leading to occlusion and flow limitation in the upper airway are identical to those corresponding to Zones I and II for the pulmonary vasculature, respectively (57).
} 
be similar to those during nasal breathing, despite reversing of the direction of airflow and the pressures upstream and downstream to the site of upper airway collapse.

\section{METHODS}

\section{Subjects}

Six subjects were drawn from the pool of tracheostomized patients studied at the Johns Hopkins Sleep Disorders Center $(n=5)$ and from the Phillips-University Marburg Sleep Disorders Center $(n=1)$. The patients' anthropometric and polysomnographic characteristics, pulmonary function, and arterial blood-gas data are detailed in Table 1. Patients were eligible if obstructive sleep apnea were present, as defined by an apnea-hypopnea index of $>5$ per hour during non-rapid eye movement (NREM) sleep on an overnight sleep study and were excluded if they currently had clinical evidence of cardiopulmonary decompensation or a previous history of upper aerodigestive tract surgery for conditions unrelated to sleep apnea. In all, six patients met selection criteria and agreed to participate in this study. In this patient sample, tracheostomy was utilized to treat their apnea because nasal continuous positive airway pressure (CPAP) was either poorly tolerated (patients $R D, R S, D I, M F, M D$ ) or ineffective at high levels (patient $S C$ ). This study was approved by the human subjects investigational review board at both institutions, and informed consent was obtained from each patient.

\section{Experimental Techniques}

Experimental apparatus. Pressure was controlled at the nose (Pn) or trachea (Ptr) over a range from -5 to $20 \mathrm{cmH}_{2} \mathrm{O}$, as previously described (40). In brief, both a positive pressure source (BiPAP, Respironics, Murryville, PA) and a negative source (modified REM-Star, Respironics) were connected to a valve that could be manually switched from one source to the other. The outflow from this valve was connected in series to a pneumotachometer (model 3700A, Hans Rudolph, Kansas City, MO) and either to a nasal mask or to the patient's tracheostomy tube. Patients were each fit with a no. 6 Shiley cuffless tracheostomy tube (6.4 mm ID, $10.8 \mathrm{~mm}$ OD, $76 \mathrm{~mm}$ length; Mallinckrodt, Hazelwood, MO).

Polysomnography. Standard polysomnographic monitoring was performed during all study protocols and included monitoring of electroencephalograms $\left(\mathrm{C}_{3}-\mathrm{A}_{2}, \mathrm{C}_{3}-\mathrm{O}_{1}\right)$, left and right electrooculograms, submental electromyogram (EMG), and electrocardiogram (modified V2 lead). Oxygen saturation was also monitored (Biox 3700, Ohmeda, Boulder, CO). Body position was monitored visually with infrared video cameras so that patients could be maintained supine throughout the protocol.

Respiratory monitoring. Tidal airflow was monitored with a pneumotachometer affixed to a tight-fitting full-face mask (during tracheostomy breathing) or to a nasal mask (during nasal breathing). Pn was monitored through a side hole in either the nasal or face mask, and Ptr was monitored through a port in the tracheostomy tube cap (during nasal breathing)

Table 1. Patient anthropometric, pulmonary function, and polysomnographic characteristics

\begin{tabular}{|c|c|c|c|c|c|c|c|c|}
\hline & \multicolumn{8}{|c|}{ Patient } \\
\hline Gender & M & $\mathrm{M}$ & M & M & $\mathrm{F}$ & M & & \\
\hline Height, cm & 165 & 173 & 175 & 183 & 162 & 189 & 174.5 & 10.3 \\
\hline Weight, kg & 128 & 171 & 124 & 99 & 105 & 140.6 & 127.9 & 26.1 \\
\hline BMI, kg/m² & 47.0 & 57.1 & 40.5 & 29.6 & 40.0 & 39.4 & 42.3 & 9.2 \\
\hline Tracheostomy duration, mo & 8 & 48 & 63 & 9 & 81 & 2 & 35.2 & 33.4 \\
\hline $\mathrm{PCO}_{2}$, Torr & 38 & 41 & 41 & 40 & 44 & 37 & 40.2 & 2.5 \\
\hline DLCO actual & 22.2 & 33.1 & & & & 16.0 & 23.8 & 8.7 \\
\hline $\mathrm{DL}_{\mathrm{CO}}, \%$ predicted & 26.2 & 31.1 & & & & 31.8 & 29.7 & 3.1 \\
\hline $\mathrm{FEV}_{1}$ actual, liters & 2.94 & 2.81 & 3.23 & 4.17 & 1.50 & 2.19 & 2.81 & 0.91 \\
\hline $\mathrm{FEV}_{1}$ predicted, liters & 3.46 & 4.24 & 3.43 & 4.56 & 1.95 & 4.23 & 3.65 & 0.95 \\
\hline FVC actual, liters & 3.89 & 3.78 & 4.22 & 5.43 & 1.86 & 2.75 & 3.66 & 1.23 \\
\hline FVC predicted, liters & 4.23 & 4.92 & 4.20 & 5.42 & 2.31 & 5.18 & 4.38 & 1.13 \\
\hline REM, \%TST & 0.0 & 5.4 & 12.0 & 18.7 & 0.0 & 0.0 & 6.0 & 7.8 \\
\hline NREM AHI, no./h & 122.5 & 100.4 & 67.5 & 72.4 & 17.7 & 104.9 & 80.9 & 37.2 \\
\hline Average base $\mathrm{SaO}_{2}$ & 96.1 & 99.2 & 98.3 & 96.0 & 89 & 92.6 & 95.2 & 3.8 \\
\hline Average low $\mathrm{Sa}_{2}$ & 77.5 & 83.8 & 81.7 & 84.0 & 78 & 83.0 & 81.3 & 2.9 \\
\hline$\%$ Obstructive & 96.0 & 94.3 & 100.0 & 100.0 & 100.0 & 100.0 & 98.4 & 2.6 \\
\hline REM AHI, no./h & $*$ & 89.5 & 40.0 & 0.0 & $*$ & $*$ & 43.2 & 44.8 \\
\hline Average base $\mathrm{SaO}_{2}$ & * & 97.7 & 93.7 & 96.0 & * & * & 95.8 & 2.0 \\
\hline Average low $\mathrm{Sa}_{2}$ & $*$ & 79.5 & 46.7 & & $*$ & $*$ & 63.1 & 23.2 \\
\hline$\%$ Obstructive & $*$ & 100.0 & 67.0 & & $*$ & $*$ & 83.5 & 23.3 \\
\hline
\end{tabular}

M, male; F, female; BMI, body mass index; tracheostomy duration, time elapsed from institution of tracheostomy to tension-time integral; DLCO, lung CO-diffusing capacity; $\mathrm{FEV}_{1}$, forced expiratory volume in $1 \mathrm{~s}$; FVC, forced vital capacity; TST, total sleep time; SE, sleep efficiency = TST/time in bed; NREM, non-rapid eye movement; REM, rapid eye movement; NREM and REM \%TST, time spent in NREM and REM sleep, respectively, as \%TST; AHI, apnea-hypopnea index, closed tracheostomy; average base and low $\mathrm{SaO}_{2}$, average baseline and average low oxyhemoglobin saturations during apneas/hypopneas, respectively; \%obstructive, \%obstructive apneic and hypopneic episodes. *Absence of REM sleep. 
and through a side hole at the outlet of the tracheostomy tube (during tracheostomy breathing). Supraglottic pressure was measured with a water-filled catheter placed transnasally into the esophagus that had a perforation at the supraglottic space. Correct placement was verified by visually inspecting the catheter's position in the oropharynx by using a small endoscope. All pressures were monitored with GouldStatham transducers (P23ID, Gould Electronics, Cleveland, $\mathrm{OH})$.

Genioglossal EMG. Fine-wire needle electrodes were utilized to monitor genioglossal activity (36). The raw signal was filtered from 30 to $3,000 \mathrm{~Hz}$ and rectified, and a moving average was computed with a time constant of $100 \mathrm{~ms}$ (Windaq/200 software, Dataq Instruments, Akron, OH).

Data acquisition. All pressures, flows, and polysomnographic parameters were amplified and recorded continuously on a polygraph recorder (Grass recorder, Astromed, Warwick, RI). Signals from analog amplifiers were also digitized at $100 \mathrm{~Hz}$ and stored on optical disk for off-line analysis (DI-200 A/D board and Windaq/200 software, Dataq Instruments).

\section{Experimental Approach}

To determine Pcrit values during tracheostomy breathing, sleep apnea patients were allowed to breathe tidally through their tracheostomies. CPAP was then applied to the tracheostomy. Breathing in this experimental condition differed from the nasal breathing route in two respects. First, the upper airway was no longer exposed to the tidal negative pressure swings in the thorax. Second, the Ptr became the Pus and the Pn became the Pds to the upper airway, as illustrated in Fig. 1. Ptr was then systematically varied through the positive-pressure range, thereby generating an outgoing flow of air through the upper airway throughout the respiratory cycle. Because the Pds remained constant and atmospheric, the level of flow venting out the upper airway solely depended on the level of pressure applied at the trachea and on the characteristics of the upper airway. We reasoned that, if Pcrit remained greater than atmospheric during tracheostomy breathing, a flow-limited condition would be obtained, as previously described $(30,32,57)$.

To verify that a flow-limited condition existed during tracheostomy breathing, we examined the effect of altering the downstream Pn on upper airway flow while a positive pressure was applied upstream at the trachea in a separate trial in two patients. Ptr $\left(20 \mathrm{cmH}_{2} \mathrm{O}\right)$ was applied while Pn was altered, either below or above Pcrit (Fig. 2). As noted above, we predicted that a change in Pn would alter the level of airflow if the upper airway were nonflow limited (i.e., $\mathrm{Ptr}>$ Pn $>$ Pcrit), whereas, during flow limitation (Ptr $>$ Pcrit $>$ Pn), a change in Pn would not change the level of airflow through the upper airway. The level of end-expiratory airflow at the tracheostomy and the mean airflow throughout the entire respiratory cycle were then calculated at each pressure level to determine whether upper airway flow had changed with alterations in Pn. In Fig. 2, a recording example is given from a patient with a Pcrit of $9.5 \mathrm{cmH}_{2} \mathrm{O}$ (patient $S C$ ) while $\mathrm{Ptr}$ was held at $22 \mathrm{cmH}_{2} \mathrm{O}$. Step changes in $\mathrm{Pn}$ from $5 \mathrm{cmH}_{2} \mathrm{O}$ to atmospheric pressure (Fig. $2 A ; \mathrm{Pn}<\mathrm{Pcrit}$ ) and from 15 to $10 \mathrm{cmH}_{2} \mathrm{O}$ (Fig. $2 B$; Pn $>$ Pcrit) were not associated with changes in airflow in the former flow-limited condition but were associated with changes in flow in the latter nonflow-limited condition. Specifically, the level of mean and end-expiratory airflow was not altered when Pn was varied below Pcrit (Fig. $2 A$ ), but it changed significantly relative to horizontal dashed lines, flow signal, when Pn was varied above Pcrit (Fig. 2B). The finding that upper airway flow did not change when Pn was less than Pcrit indicated that a flow-limited condition was obtained, because upper airway flow was independent from the downstream Pn. Similar findings were also demonstrated in the second patient studied. We conclude that the upper airway remained in a
Fig. 2. Recording example from $p a-$ tient $S C$ illustrating pressure-flow signals at specific levels of downstream Pn while the upstream Ptr remained constant at $20 \mathrm{cmH}_{2} \mathrm{O}$ during rapid eye movement sleep. Note that airflow was measured at the tracheostomy (ivtr) and that the inspiratory flow contour paralleled respiratory effort during rapid eye movement sleep rather than a reflection of the upper airway properties. When Pn was less than Pcrit $(A)$, a step change in Pn did not alter the level of end-expiratory or mean flow throughout the entire respiratory cycle, whereas when Pn was greater than Pcrit $(B)$, the level of both endexpiratory and mean flow changed significantly with the step changes in Pn (see horizontal dashed lines in flow signals). The finding indicated that the upper airway remained in the flow-limited state whenever the downstream Pn was lower than Pcrit. EOG, electroocculogram; EEG, electroencephalogram; $\mathrm{EMG}_{\mathrm{GG}}$, electromyogram of the genioglossus muscle. See text for details.

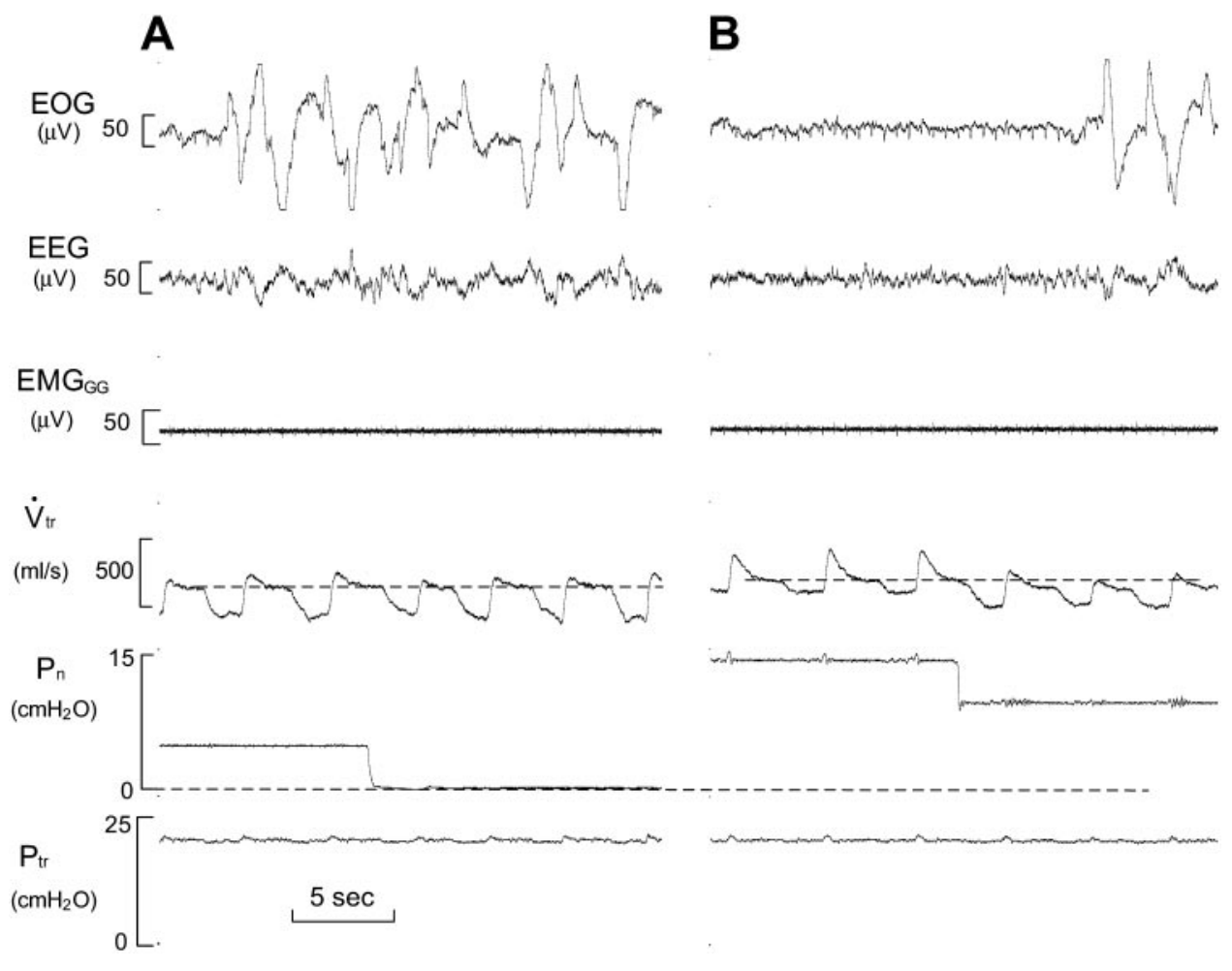

J Appl Physiol • VOL 93 • OCTOBER 2002 • www.jap.org 
flow-limited state as long as Pn remained atmospheric and that upper airway pressure-flow relationships could be generated by altering the Ptr.

During the steady-state application of positive pressure, pressure-flow relationships were constructed from measurements of upper airway flow and Ptr at specific points in the respiratory cycle. As previously described for pressure-flow relationships during nasal breathing, a linear relationship was predicted between the level of airflow venting out the upper airway [maximal airflow $\left(\dot{\mathrm{V}}_{\max }\right)$ ] and the Ptr applied (10), once the upstream Ptr exceeded a Pcrit. These relationships were then analyzed to determine $(43,49)$ Pcrit values at peak expiration, end expiration, and peak inspiration (see below).

To assess Pcrit differences between nasal and tracheostomy breathing, pressure-flow relationships were also generated while patients inspired through their upper airway after their tracheostomies were capped. Under these circumstances, the Pn was now considered to be upstream and the Ptr downstream during inspiration, as previously described (10). The Pcrit was determined by altering $(43,44,49)$ the upstream Pn experimentally while monitoring inspiratory airflow (40). At each level of upstream Pn, flow-limited breaths were defined by a plateauing of inspiratory airflow at a maximal level ( $\dot{V}_{\text {max }}$ ) as the downstream Ptr fell progressively during inspiration (44, 49). From these flow-limited breaths, Pn and $\dot{V}_{I_{\text {max }}}$ values were taken at peak inspiration as defined by the nadir of Ptr swings. Pressure-flow data obtained from these flow-limited inspirations were then used to define the relationship of $\dot{V}_{I_{\max }}$ vs. Pn from which the Pcrit was derived (40). Pcrit values derived from the Pn-flow relationship during inspiration and from Ptr-flow relationships throughout the respiratory cycle were subsequently compared to examine the influence of respiratory phase and airflow regimen on upper airway collapsibility during sleep.

\section{Experimental Protocols for Altering Pus}

During each experiment, the patient slept supine with the head supported by one pillow. Head and neck position was maintained constant throughout the experiments. The Pus was varied at the nose or in the trachea (Fig. 1) as follows.

Experiment 1: Altering Ptr. The tracheostomy tube was opened, and positive pressure was initially applied to the tube. A holding Ptr was then established by increasing the level of Ptr stepwise until the level of airflow exiting the upper airway exceeded $300 \mathrm{ml} / \mathrm{s}$. The mean holding Ptr for the group was $15.5 \pm 2.3(\mathrm{SE}) \mathrm{cmH}_{2} \mathrm{O}$. Ptr was then lowered repeatedly until upper airway flow ceased during stable NREM sleep (see Fig. 1A). With each Ptr drop, Ptr was held for up to five breaths to allow the upper airway flow to reach a steady-state level.

Experiment 2: Altering Pn. With the tracheostomy tube capped, Pn was first set to a holding pressure level at which upper airway obstruction was abolished, as previously described $(6,40)$. The mean holding Pn for the group was $13.8 \pm$ $2.4(\mathrm{SE}) \mathrm{cmH}_{2} \mathrm{O}$. After the holding pressure was maintained for at least $3 \mathrm{~min}$ of stable NREM sleep, Pn was then abruptly lowered for five breaths or until $20 \mathrm{~s}$ of apnea or an arousal had occurred (see Fig. 1B). With each Pn drop, Pn was maintained for up to five breaths to allow the level of $\dot{\mathrm{V}}_{\mathrm{I}_{\text {max }}}$ to reach a steady-state level (6). Pn was then increased to the holding level for $45-60 \mathrm{~s}$ and was then repeatedly lowered in $\sim 2-\mathrm{cmH}_{2} \mathrm{O}$ decrements until airflow ceased (obstructive apnea occurred). Thereafter, the series was repeated until a total of 10-15 runs had occurred. If arousal occurred, the patient was allowed to reinitiate stable NREM sleep before continuing the protocol.

\section{Data Analysis}

Analysis of pressure-flow relationships. Pressure-flow relationships were constructed from breaths obtained during step reductions in the Pus (Ptr or Pn) (6). Ptr-flow relationships were generated from levels of airflow venting out the upper airway at specific points in the respiratory cycle (experiment 1). The Pn-flow relationship was constructed from levels of $\dot{\mathrm{V}}_{\text {max }}$ as the upstream Pn was varied (experiment 2). Each relationship was constructed from data obtained from steady-state levels of airflow in breaths after abrupt decreases in Pus, as described below.

$\dot{\mathrm{V}}_{\text {MAX }}$ VS. PTR RELATIONSHIP. During step decreases in Ptr, the level of airflow through the upper airway increased over the first two to three breaths to a steady-state level. Once a steady state was established in airflow, airflow was measured at discrete points in the respiratory cycle for the next two to three breaths as follows. The inspiratory time point was measured at the nadir in Ptr, and the peak and endexpiration points were measured at the peak Ptr and endexpiratory plateau, respectively. At each time point, airflow and Ptr were measured for each breath analyzed, and separate pressure-flow relationships were constructed for each point in the respiratory cycle. Each relationship was analyzed separately by least squares linear regression. The Pcrit and resistance upstream to the site of upper airway collapse (Rus) were given by the level of Ptr below which airflow ceased and by the reciprocal of the slope of the pressure-flow relationship, as previously described $(43,49)$.

$\dot{\mathrm{V}}_{\text {MAX }}$ VS. PN RELATIONSHIP. During step decreases in Pn, the level of $\dot{V} I_{\max }$ declined to a steady-state level by the third breath, as previously described (43). The third through fifth breaths during Pn drops were examined for the presence of inspiratory airflow limitation, as previously described (43, 49). In flow-limited inspirations, levels of $\dot{V}_{I_{\max }}$ and Pn were tabulated, and least squares linear regression was employed to describe the pressure-flow relationship (Minitab, State College, PA). From each relationship, the Pcrit and Rus were computed, as described above.

Genioglossal EMG. The tonic, peak, and phasic (peak minus tonic) moving-average EMG activities were measured for steady-state breaths after step reductions in Pus to the Pcrit level for both the nasal and tracheal breathing route. The EMG was measured for the third through fifth breaths after a drop in Pus, as noted in the analysis of pressure-flow relationships above.

Statistical analysis. Paired two-tailed $t$-tests and generalized linear regression (fixed factors: nasal vs. tracheal breathing and respiratory phase; random factor: patient code) were utilized to assess for differences in Pcrit and Rus (Minitab). Bonferroni's post hoc comparisons were performed to determine the source of significance when significant differences were found by the generalized linear regression procedure. EMG data were analyzed by generalized linear regression (fixed factor: nasal vs. tracheal breathing; random factors: patient code and breath number) to assess for differences in tonic and phasic activity (Minitab). A $P<0.05$ was considered significant.

\section{RESULTS}

\section{Effect of Altering Pus on Upper Airway Flow}

Ptr-flow recordings. In Fig. 3, the effect of lowering Pus at the trachea (Fig. $3 A$ ) on upper airway flow is 




illustrated for one patient (patient DI). During tracheostomy breathing, pressure applied to a patient's tracheostomy was lowered stepwise from 19.6 to 12.4 and $8.1 \mathrm{cmH}_{2} \mathrm{O}$. Two major findings are illustrated in these recordings. First, the level of air flowing out the upper airway varied in relation to the Ptr level. At higher (more positive) Ptr, a continuous flow of air was observed through the upper airway (Fig. 3A, left and middle), whereas no flow was observed at the lowest Ptr applied (Fig. 3A, right). Of note, upper airway flow ceased when the Ptr was still positive, indicating that the upper airway Pcrit was in the positive range. Moreover, the supraglottic pressure also remained positive and tracked the level of Ptr applied, indicating that this pressure was also upstream to the site of upper airway occlusion. This latter finding suggested that the pharynx was the site of upper airway occlusion and that its Pcrit was positive, as predicted by the Starling resistor model (see Background).

Second, CPAP abolished the negative pressure swings in the trachea during inspiration. Rather, Ptr remained positive and varied only minimally throughout the respiratory cycle. Under these circumstances, the upstream Ptr remained greater than the Pcrit, which was greater than the downstream Pn (atmospheric), indicating that the upper airway remained in a flow-limited condition (Pus > Pcrit > Pds; see Background). During flow limitation, tidal fluctuations in airflow occurred throughout the respiratory cycle (see Fig. 3A, left and middle), suggesting phasic alterations in upper airway function (see below).

Pn-flow recordings. In Fig. 3, pressure-flow recordings are illustrated for the same patient breathing through his upper airway at a $\mathrm{Pn}$ of $8.0 \mathrm{cmH}_{2} \mathrm{O}$ with his tracheostomy tube capped (Fig. $3 B$ ). In contrast to tracheostomy breathing at a comparable level of Ptr (Fig. 3A, right), airflow did not cease at this level of Pn. Rather, considerable upper airway flow was observed. The marked inspiratory Ptr and supraglottic pressure swings and the plateauing of inspiratory airflow indicated that a flow-limited condition was obtained only during mid-inspiration, as previously described $(6,43)$. Of note, the upper airway occluded at a considerably lower Pn vs. Ptr level in this patient (0.8 vs. 8.1 $\mathrm{cmH}_{2} \mathrm{O}$ ), indicating that Pcrit differed markedly during tracheostomy compared with nasal breathing.

\section{Pressure-Flow Relationships During Nasal and Tracheostomy Breathing}

To account for variability in pressure and flow throughout the respiratory cycle, separate upper airway pressure-flow relationships were constructed for peak inspiration, peak expiration, and end expiration during tracheostomy breathing in Fig. $4 A$ (patient SC). Confirming the prediction of the Starling resistor model, we found that the pressure-flow relationships describing each phase of the respiratory cycle were characterized by the cessation of upper airway flow when Ptr fell below a Pcrit, and by a linear relationship between airflow and Ptr when Ptr exceeded the Pcrit. Comparing the pressure-flow relationships in this patient between peak expiration and peak inspiration, we observed that the Pcrit values were somewhat higher and the slopes were steeper at the peak expiratory time point, suggesting that the Pcrit increased and the Rus decreased during expiration.

In Fig. $4 B$, the Pn-flow relationship is illustrated for the same patient (patient $S C$ ). As noted above for the Ptr-flow relationship, the nasal relationship was linear and was characterized by closure of the upper airway (zero airflow) when the upstream Pn fell below a Pcrit. Despite these similarities, a marked leftward shift in the Pn-flow relationship can be discerned compared with the tracheal relationship. This shift is primarily explained by a reduction in Pcrit during nasal breathing (comparing Pn vs. Ptr at the zero-flow point), indi- 


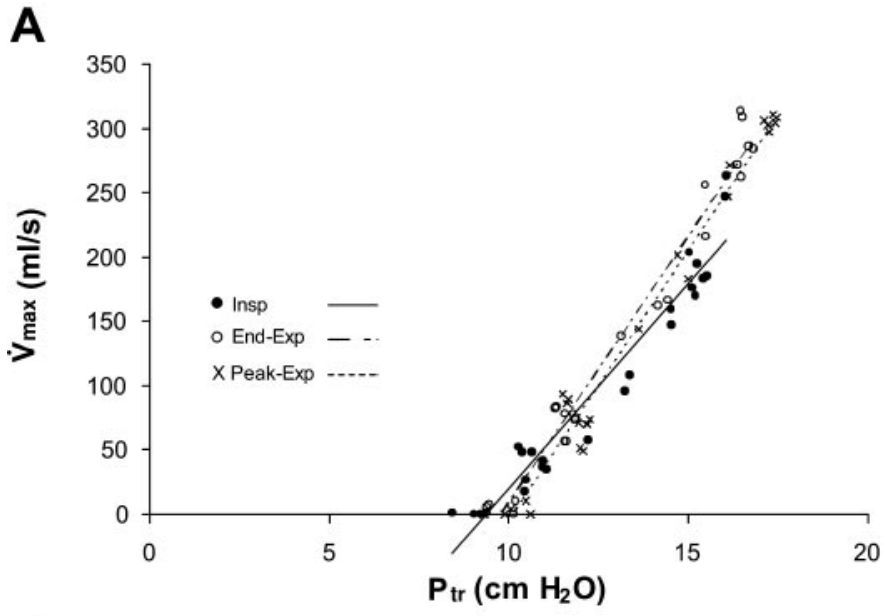

B

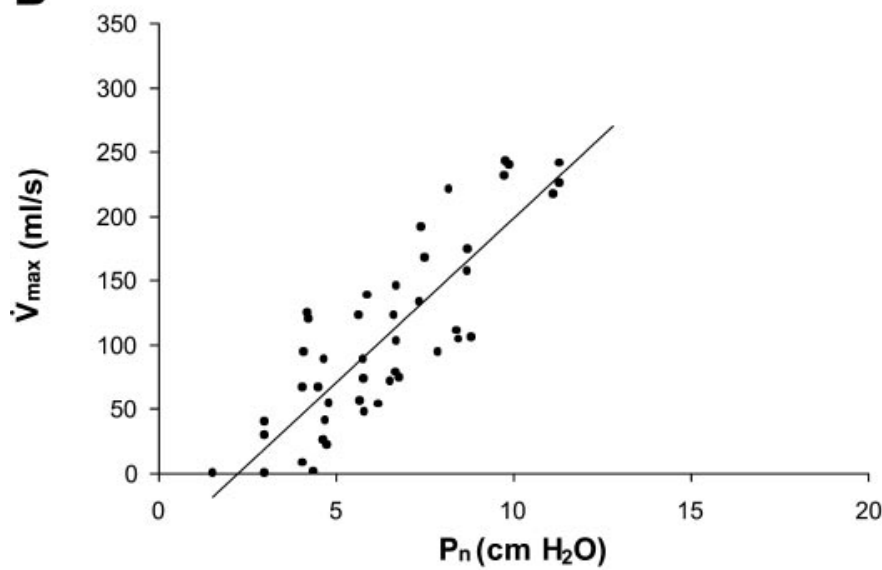

Fig. 4. Pressure-flow relationships are shown for patient $S C$. Vua vs. Pus is graphed when Ptr was varied $(A)$ and when Pn was varied $(B)$. Least squares linear regression lines are also plotted for data points used to construct each pressure-flow relationship. $\dot{\mathrm{V}}_{\max }$, maximal airflow. A: X, tracheal peak expiratory (peak-exp); $\bigcirc$, end expiratory (end-exp); •, peak inspiratory (insp). B: •, nasal. Note that all Ptr-flow relationships are shifted to the right of the nasal relationship.

cating diminished upper airway collapsibility during nasal vs. tracheostomy breathing in this patient.

\section{Modulation of Pcrit During Sleep}

Effect of respiratory phase on Pcrit. The influence of respiratory phase on Pcrit values is illustrated in Fig. 5 (comparison A) during tracheostomy breathing. A small but significant decrease in Pcrit from $7.1 \pm 1.6$ to $6.6 \pm 1.9$ to $6.1 \pm 1.9 \mathrm{cmH}_{2} \mathrm{O}$ was observed from peak expiration through end expiration to peak inspiration, respectively (means $\pm \mathrm{SE}, P<0.05$ ). In contrast, progressive increases in Rus were observed from $30.4 \pm 17.6$ to $35.0 \pm 18.2$ (at end expiration) to $38.6 \pm$ $14.2 \mathrm{cmH}_{2} \mathrm{O} \cdot 1^{-1} \cdot \mathrm{s}$ at peak inspiration (means $\pm \mathrm{SE}$, $P<0.05)$.

Effect of airflow regimen on Pcrit. The effect of differences in airflow regimen on Pcrit is illustrated for the nasal and tracheostomy breathing routes at peak inspiration (Fig. 5, comparison B). In this comparison, we detected a significantly lower Pcrit during nasal $\left(1.1 \pm 1.5 \mathrm{cmH}_{2} \mathrm{O}\right)$ vs. tracheostomy $\left(6.1 \pm 1.9 \mathrm{cmH}_{2} \mathrm{O}\right)$ breathing (means $\pm \mathrm{SE}, P<0.01$ ), indicating that upper airway collapsibility fell markedly when patients breathed nasally. In contrast, no significant difference in Rus was detected between these two conditions (nasal, $35.8 \pm 17.6 \mathrm{cmH}_{2} \mathrm{O} \cdot 1^{-1} \cdot \mathrm{s}$; tracheal, $38.6 \pm$ $\left.14.2 \mathrm{cmH}_{2} \mathrm{O} \cdot 1^{-1} \cdot \mathrm{s}\right)$ for the group as a whole.

Airflow regimen and site of collapse. To determine whether alterations in the site of upper airway obstruction (laryngeal vs. pharyngeal) accounted for Pcrit differences between nasal and tracheostomy breathing, we monitored pressure in the supraglottic space in two patients (patients $D I$ and $R D$ ) during the application of steady-state levels of positive pressure at either the tracheostomy or nose (Fig. 3, patient DI). During periods of complete upper airway occlusion (absent airflow, Fig. 3A, right), tidal excursions in the supraglottic pressure paralleled those in the trachea, and a pressure gradient was observed between the supraglottic space and nasal opening in both patients. A similar divergence between $\mathrm{Pn}$ and supraglottic pressures was observed during nasal breathing in both patients (not shown). These findings indicated that the pharynx remained the site of upper airway obstruction during both nasal and tracheostomy breathing.

\section{Genioglossal EMG Activity and Tidal Ptr Swings}

To further delineate the mechanism for Pcrit differences between tracheostomy vs. nasal breathing, genioglossal EMG and tidal swings in Ptr were measured in three subjects (patients $M F, R D$, and $S C$ ) during periods of complete upper airway occlusion in each condition. Both the tonic and peak phasic genioglossal

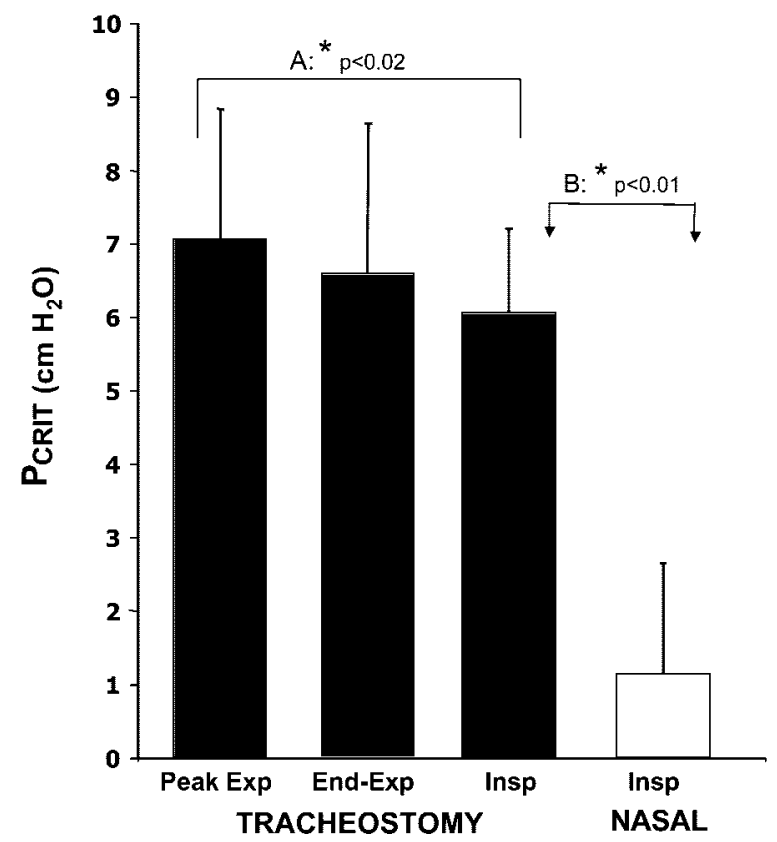

Fig. 5. Effect of nasal (open bar) vs. tracheostomy (solid bars) breathing and respiratory phase (tracheostomy breathing: peak expiratory, end expiratory, and inspiratory) on Pcrit is illustrated. Comparisons $A$ and $B$ are illustrated by brackets and arrows. Values are means $\pm \mathrm{SE}$. See text for details. 
EMG activity were found to be higher during nasal vs. tracheostomy breathing (Fig. $6, P<0.0001$ ). During tracheostomy breathing, phasic activity was significantly higher than tonic activity (Fig. $6, P<0.0001$ ). Tracheostomy breathing was also associated with markedly reduced tidal swings in $\operatorname{Ptr}(2.0 \pm 0.4$ vs. $25.1 \pm 15.3 \mathrm{cmH}_{2} \mathrm{O} ; P=0.06$ ). These findings suggest that reflex responses to negative Ptr may account for differences in Pcrit with alterations in respiratory phase and airflow regimen.

\section{DISCUSSION}

In the present study, the dynamic modulation of upper airway function was examined during tidal breathing in sleeping patients with obstructive sleep apnea. A novel method was developed for making multiple determinations of the Pcrit and Rus from upper airway pressure-flow relationships in tracheostomized patients. Three major findings resulted from the analysis of Pcrit values in the isolated upper airway segment in sleeping apneic patients. First, we found that the upper airway occluded when the upstream Ptr was positive, confirming Starling resistor behavior for the upper airway with a positive Pcrit during tracheostomy breathing. Second, during tracheostomy breathing, a small yet significant decrease in Pcrit was found at the inspiratory vs. peak and end-expiratory time points (comparison A). This second finding indicated that the Pcrit was modulated by phasic factors during tidal breathing. Third, we found that the inspiratory Pcrit was markedly lower during nasal vs. tracheostomy breathing (comparison $B$ ), indicating that the Pcrit varied substantially with alterations in airflow regimen. Our findings indicate that both phasic factors

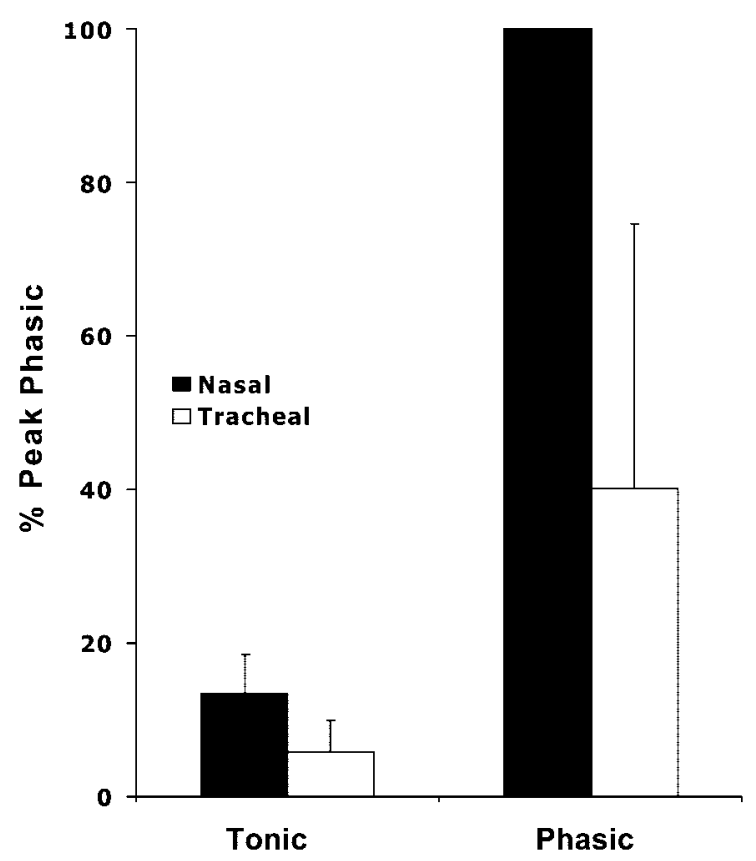

Fig. 6. Tonic and phasic $\mathrm{EMG}_{\mathrm{GG}}$ activity (expressed as \%peak phasic activity) during nasal and tracheostomy breathing. Values are means $\pm \mathrm{SE}$. and airflow regimen play a role in modulating upper airway collapsibility dynamically during tidal breathing in sleeping apneic patients and imply that differences in airflow regimen between inspiration and expiration lead to marked tidal alterations in upper airway collapsibility.

\section{Modeling Upper Airway Function During Tracheostomy Breathing}

A major finding of the present study was that Ptrflow relationships were remarkably similar to those previously demonstrated for the upper airway during nasal breathing $(10,40,43,44,49)$ and for other collapsible biological conduits $(29,57)$ in two respects. First, airflow ceased (the upper airway occluded) when the upstream Ptr fell below the Pcrit. Upper airway occlusion was confirmed by the fact that a pressure gradient persisted between the Pus and Pds (Pcrit $>$ Ptr $>$ Pn $=0$ ). Second, airflow vented out the upper airway as the upstream Ptr exceeded the Pcrit. Under these circumstances, a flow-limited condition ensued for the upper airway because Pcrit values were uniformly positive in our patients relative to atmospheric pressure downstream at the nose (Ptr $>$ Pcrit $>\mathrm{Pn})$ (see Effect of airflow regimen on Pcrit) $(51,57)$. In addition, a linear Ptr-flow relationship was observed once the Ptr exceeded the Pcrit, as predicted by the Starling resistor model. As with Pn-flow relationships, therefore, our findings during tracheostomy breathing now indicate that Ptr-flow relationships are characteristic of those in a Starling resistor $(6,9,10,43,44,49)$ in sleeping apneic patients and provide direct evidence for flow limitation during expiration when air vents out of the upper airway to atmosphere.

We utilized tracheostomy breathing to model upper airway flow dynamics throughout the respiratory cycle. In contrast to pressure-flow relationships in an unobstructed airway, the Ptr-flow relationship was shifted markedly to the right (see Fig. 4), indicating that the upper airway remained occluded at positive Ptr levels. This finding, reflecting a positive Pcrit during tracheostomy breathing, is consistent with previous reports of spontaneous upper airway occlusion during complete neuromuscular blockade $(13,14)$ and occlusion during expiration $(2,3,25)$ when Ptr values are also known to be positive. Our findings now confirm these previous observations and extend them by characterizing the entire pressure-flow relationship throughout the respiratory cycle in spontaneously breathing sleeping apneic patients. Moreover, the Pcrit values derived in these patients address a fundamental question regarding the pathogenesis of upper airway occlusion in obstructive sleep apnea. They imply that a positive Pcrit is required for the development of upper airway occlusion in this disorder $(17,43,49)$, rather than negative Ptr values during inspiration, as previously suggested (33). In fact, the markedly negative inspiratory Ptr values generated during obstructive apneic episodes appear to be the consequence instead of the cause of upper airway occlusion. Thus our analysis 
of pressure-flow relationships during tracheostomy breathing now indicates that upper airway collapsibility is markedly elevated during tracheostomy vs. nasal breathing. Moreover, the airflow regimen during tracheostomy breathing resembles that normally present during expiration, suggesting that the upper airway further obstructs expiratory airflow in sleeping apneic patients.

\section{Mechanism for Upper Airway Obstruction During Tracheostomy Breathing}

Effect of respiratory phase. In this study, we examined upper airway pressure-flow relationships throughout the respiratory cycle during tracheostomy breathing. Our analysis indicated that the Pcrit fell modestly from peak expiration through inspiration (comparison A), whereas the Rus rose progressively. Although the explanation for reciprocal changes in Pcrit and Rus is not clear, such changes may be best attributed to increases in airway axial tension as tracheal and laryngeal structures are pulled caudally by the diaphragm during inspiration $(34,53,55,56)$. Alternatively, observed decreases in Pcrit and increases in Rus may have been related to the separate actions of pharyngeal dilator and constrictor muscles, respectively, that are known to activate phasically $(18-20,23,37)$. Thus observed changes in Pcrit and Rus could have been due to phasic alterations in upper airway neuromuscular activity and/or mechanical traction by caudal structures throughout the respiratory cycle. Regardless of the mechanism for these changes, our findings indicate that phasic mechanisms played some role in modulating upper airway collapsibility.

Effect of airflow regimen. Further insight into the mechanism for elevations in Pcrit during tracheostomy breathing can be gained from our comparison of Pcrit values between nasal and tracheostomy breathing (comparison B). A major finding was that Pcrit values were consistently higher during tracheostomy than nasal breathing throughout the respiratory cycle (see Fig. 5), an effect that can best be attributed to differences in the airflow regimen between the two breathing routes. In previous human and animal studies, such elevations in Pcrit have been related to decreases in upper airway neuromuscular activity $(7,28,31,35$, $45,46)$. In fact, marked reductions in peak phasic and tonic neuromuscular activity have been demonstrated in awake subjects during tracheostomy breathing (23) and have been attributed to alterations in reflex responses to negative pressure $(1,5,12,16,24,47)$ and/or lung inflation $(8,24,57)$. We found similar decreases in genioglossal activity in our patients during tracheostomy breathing. Of note, the further decrease in upper airway neuromuscular activity during tracheostomy breathing is particularly noteworthy because it fell from an already relatively hypotonic level during nasal breathing (40). Thus our findings indicate that a diminution in neuromuscular activity could account for the marked elevations in Pcrit observed during tracheostomy vs. nasal breathing and suggest that the upper airway is even more markedly hypotonic during tracheostomy breathing.

Alternatively, observed differences in upper airway function may have been simply due to a change in the direction of airflow through the upper airway rather than differences in neuromuscular control between nasal and tracheal breathing routes. It is possible that elevations in Pcrit were related to alterations in the site of upper airway collapse during tracheostomy vs. nasal breathing. Although our manometric measurements established a pharyngeal site of collapse (Fig. 3, see Effect of airflow regimen on Pcrit), we did not further localize this site within the pharynx. Nevertheless, investigators have previously determined that the velopharynx is the most common site of collapse, particularly under conditions of reduced or absent upper airway neuromuscular activity $(15,26)$. Because our patients also exhibited hypotonia during tracheostomy breathing, we believe the velopharynx to have been the most likely site of primary collapse during both nasal and tracheostomy breathing. If switching to tracheostomy breathing led to even more marked elevations in the velopharyngeal Pcrit, however, the velopharynx would have occluded more readily, thereby forcing air to discharge out the mouth. In fact, investigators have observed endoscopically that the soft palate occasionally billows up against the posterior pharyngeal wall during expiration (S. Isono, personal communication), effectively sealing off the nasopharynx. Under these circumstances, our Pcrit measurements would have reflected oropharyngeal rather than velopharyngeal Pcrit values. Because oropharyngeal Pcrit values are known to be considerably lower (15), we may have

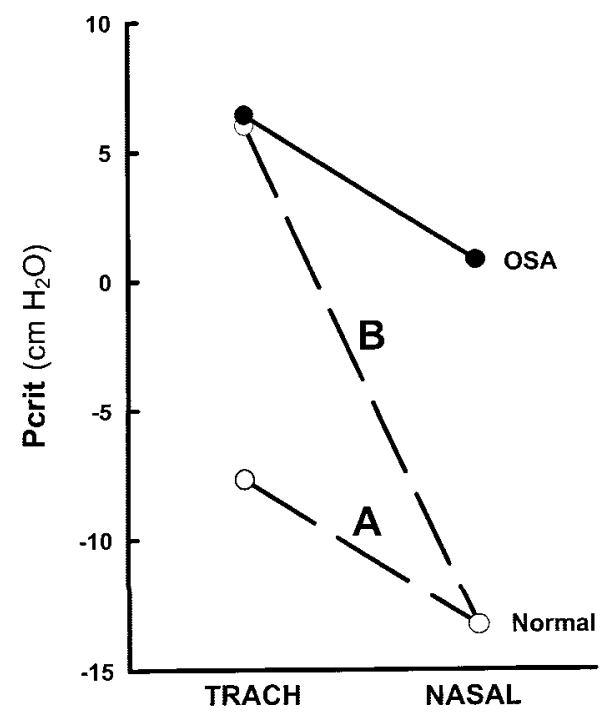

Fig. 7. Effect of breathing route on Pcrit in patients with obstructive sleep apnea (OSA) and normal individuals. Mean data are represented for nasal and tracheostomy breathing routes in OSA patients from the present study and for nasal breathing in normal subjects during sleep (44). Possible Pcrit responses to changes in breathing route are illustrated for normal individuals (dashed lines) and may be similar (response A) or greater (response $B$ ) than OSA response (solid line) shown. $\bullet$, Actual data; $\bigcirc$, hypothetical data. See text for details. 
underestimated the Pcrit values between nasal and tracheostomy breathing. Therefore, observed increases in Pcrit values during tracheostomy breathing cannot be explained simply by migration of site of collapse.

\section{Limitations of the Present Study}

There are several limitations in the present study. First, the sample size was small because of the difficulty in recruiting medically stable tracheostomized patients for laborious physiological studies, leaving some question as to the generalizability of our findings. Despite our small patient sample, however, it is remarkable that consistent differences in Pcrit were still detected with alterations in breathing route and respiratory phase. Second, our observations were limited to a group of apneic patients who demonstrated upper airway obstruction during nasal breathing. It would be equally important to determine whether the modulation of Pcrit differs in normal individuals during sleep. Third, although we argue that observed changes in upper airway function were related to alterations in neuromuscular activity, we recognize that such activity was monitored in only three of our patients. We still believe that neuromuscular mechanisms account for the dynamic changes in upper airway function that we have demonstrated, because similar EMG changes have been well documented in previous studies with alterations in breathing route (23), respiratory phase (36), and upper airway obstruction $(16,40)$. In this regard, it is quite likely that even greater phasic changes in upper airway collapsibility would have been observed had our methods allowed for expiratory Pcrit measurements during nasal breathing, when phasic neuromuscular activity is known to be greater (23). Fourth, we recognize that different upper airway segments determine the Rus during nasal and tracheal breathing, thereby limiting comparisons in this parameter to phasic periods within the tracheostomy breathing condition. Nevertheless, differences in airflow regimen were associated with a marked rightward shift in the Ptr-flow relationships, clearly establishing that tracheostomy breathing was associated with a substantial rise in Pcrit.

\section{Study Implications}

There are two major implications of the present findings. First, the marked rightward shift in the pressure-flow relationship during tracheostomy breathing indicates that airflow obstruction worsens during expiration. This increase in upper airway obstruction can be attributed to elevations in Pcrit and Rus, both of which impeded the discharge of exhaled gas in our apneic patients. As the Pcrit rose, the upper airway closed prematurely (when Ptr was still well above atmospheric) and trapped exhaled gas within the lungs. In contrast, an elevated resistance would lengthen the time required to exhale completely, thereby prolonging expiration. Such increases in Pcrit and Rus might lead to static and dynamic lung hyperinflation (54), respectively, if not for reflex recruitment of expiratory muscles that raise Ptr values concomitantly (48). Our findings indicate, therefore, that the mechanical loads imposed by the upper airway on the respiratory system during expiration are even greater than those during inspiration. On the other hand, concomitant elevations in expiratory Pcrit values during this phase would help to maintain a higher lung volume during apneic episodes and thereby attenuate the oxyhemoglobin desaturation.

It is worth noting that the Pcrit fell by $>6 \mathrm{cmH}_{2} \mathrm{O}$ when patients switched from tracheostomy to nasal breathing. With decreases in Pcrit of this magnitude, significant reductions in the severity of sleep-disordered breathing have been demonstrated, depending on the absolute level to which the Pcrit falls (42). As noted above, this decrease in Pcrit in our apneic patients could have been due to increases in upper airway neuromotor tone that resulted from breathing nasally through an obstructed upper airway. Nevertheless, Pcrit values during nasal breathing in our patients remained higher than those in normal snoring and nonsnoring individuals $(9,44)$. Based on the present findings, we now offer two explanations for Pcrit differences between normal subjects and apneic patients during nasal breathing (Fig. 7). First, anatomic factors may increase upper airway collapsibility, particularly under conditions of reduced or absent upper airway neuromotor tone. Under these circumstances, we would expect to find the Pcrit to be higher in apneic patients compared with normal subjects during tracheostomy breathing but that an increase in neuromuscular tone would lead to similar decreases in Pcrit after each group switched to nasal breathing (Fig. 7, compare response $A$ in normal subjects to apneic patients, solid line). Alternatively, disturbances in neuromuscular control in apneic patients may have compromised the airway's ability to maintain airway patency during nasal breathing. If true, normal individuals would be expected to exhibit an even greater decrease in Pcrit (Fig. 7, response $B$ ) than apneic patients. In other words, a blunted response in apneic patients might reflect a loss of compensatory reflexes $(4,46)$ that could mitigate against anatomic compromise of airway patency (13). In theory, examining the influence of breathing route on Pcrit values might also allow investigators to determine the anatomic and neuromuscular basis for Pcrit elevations associated with adiposity (39). Further research will be required to delineate the impact of alterations in upper airway anatomy and disturbances in neuromuscular control on Pcrit values in apneic patients.

\footnotetext{
This study was supported by National Heart, Lung, and Blood Institute Grants HL-50381 and HL-37379 and by Deutsche Forschungsgemeinschaft SCHN543/1.
}

\section{REFERENCES}

1. Aronson RM, Onal E, Carley DW, and Lopata M. Upper airway and respiratory muscle responses to continuous negative airway pressure. J Appl Physiol 66: 1373-1382, 1989.

2. Badr MS, Kawak A, Skatrud JB, Morrell MJ, Zahn BR, and Babcock MA. Effect of induced hypocapnic hypopnea on upper 
airway patency in humans during NREM sleep. Respir Physiol 110: 33-45, 1997.

3. Badr MS, Toiber F, Skatrud JB, and Dempsey J. Pharyngeal narrowing/occlusion during central sleep apnea. J Appl Physiol 78: 1806-1815, 1995.

4. Berry RB, Kouchi KG, Bower JL, and Light RW. Effect of upper airway anesthesia on obstructive sleep apnea. Am J Respir Crit Care Med 151: 1857-1861, 1995.

5. Berry RB, McNellis MI, Kouchi K, and Light RW. Upper airway anesthesia reduces phasic genioglossus activity during sleep apnea. Am J Respir Crit Care Med 156: 127-132, 1997.

6. Boudewyns A, Punjabi N, Van de Heyning PH, De Backer WA, O'Donnell CP, Schneider H, Smith PL, and Schwartz AR. Abbreviated method for assessing upper airway function in obstructive sleep apnea. Chest 118: 1031-1041, 2000.

7. Brouillette RT and Thach BT. A neuromuscular mechanism maintaining extrathoracic airway patency. J Appl Physiol 46: 772-779, 1979 .

8. Gauda EB, Carroll TP, Schwartz AR, Smith PL, and Fitzgerald RS. Mechano- and chemoreceptor modulation of respiratory muscles in response to upper airway negative pressure. J Appl Physiol 76: 2656-2662, 1994.

9. Gleadhill IC, Schwartz AR, Schubert N, Wise RA, Permutt S, and Smith PL. Upper airway collapsibility in snorers and in patients with obstructive hypopnea and apnea. Am Rev Respir Dis 143: 1300-1303, 1991.

10. Gold AR and Schwartz AR. The pharyngeal critical pressure. The whys and hows of using nasal continuous positive airway pressure diagnostically.Chest 110: 1077-1088, 1996.

11. Horner RL, Innes JA, Morrell MJ, Shea SA, and Guz A. The effect of sleep on reflex genioglossus muscle activation by stimuli of negative airway pressure in humans. J Physiol 476: 141-151, 1994.

12. Horner RL, Innes JA, Murphy K, and Guz A. Evidence for reflex upper airway dilator muscle activation by sudden negative airway pressure in man. J Physiol 436: 15-29, 1991.

13. Isono S, Remmers JE, Tanaka A, Sho Y, Sato J, and Nishino T. Anatomy of pharynx in patients with obstructive sleep apnea and in normal subjects. J Appl Physiol 82: 13191326, 1997.

14. Isono S, Tanaka A, Remmers JE, and Nishino T. Comparison of static mechanics of passive pharynx between patients with obstructive sleep apnea and normal subjects (Abstract). Am J Respir Crit Care Med 151: A667, 1995.

15. Isono S, Tanaka A, Sho Y, Konno A, and Nishino T. Advancement of the mandible improves velopharyngeal airway patency. J Appl Physiol 79: 2132-2138, 1995.

16. Issa FG, Edwards P, Szeto E, Lauff D, and Sullivan C. Genioglossus and breathing responses to airway occlusion: effect of sleep and route of occlusion. J Appl Physiol 64: 543-549, 1988.

17. King E, Seelagy M, Schwartz A, Nadeau J, Permutt S, and Smith P. Induction of sleep apnea in normal individuals with nasal continuous negative airway pressure (Abstract). Am Rev Respir Dis 145: A409, 1992.

18. Kuna ST and Smickley JS. Superior pharyngeal constrictor activation in obstructive sleep apnea. Am J Respir Crit Care Med 156: 874-880, 1997.

19. Kuna ST, Smickley JS, and Insalaco G. Posterior cricoarytenoid muscle activity during wakefulness and sleep in normal adults. J Appl Physiol 68: 1746-1754, 1990.

20. Kuna ST, Smickley JS, and Vanoye CR. Respiratory-related pharyngeal constrictor muscle activity in normal human adults. Am J Respir Crit Care Med 155: 1991-1999, 1997.

21. Lofaso F, d'Ortho MP, Fodil R, Delclaux C, Harf A, and Lorino AM. Abdominal muscle activity in sleep apnea during continuous positive airway pressure titration. Chest 120: 390$396,2001$.

22. Lorino AM, Lofaso F, Duizabo D, Zerah F, Goldenberg F, d'Ortho MP, Harf A, and Lorino H. Respiratory resistive impedance as an index of airway obstruction during nasal continuous positive airway pressure titration. Am J Respir Crit Care Med 158: 1465-1470, 1998.

23. Malhotra A, Fogel RB, Edwards JK, Shea SA, and White DP. Local mechanisms drive genioglossus activation in obstruc- tive sleep apnea. Am J Respir Crit Care Med 161: 1746-1749, 2000 .

24. Mathew OP, Abu-Osba YK, and Thach BT. Influence of upper airway pressure changes on genioglossus muscle respiratory activity. J Appl Physiol 52: 438-444, 1982.

25. Morrell MJ, Arabi Y, Zahn B, and Badr MS. Progressive retropalatal narrowing preceding obstructive apnea. Am J Respir Crit Care Med 158: 1974-1981, 1998.

26. Morrison DL, Launois SH, Isono S, Feroah TR, Whitelaw WA, and Remmers JE. Pharyngeal narrowing and closing pressures in patients with obstructive sleep apnea. Am Rev Respir Dis 148: 606-611, 1993.

27. Neill AM, Angus SM, Sajkov D, and McEvoy RD. Effects of sleep posture on upper airway stability in patients with obstructive sleep apnea. Am J Respir Crit Care Med 155: 199-204, 1997.

28. Oliven A, Odeh M, and Gavriely N. Effect of hypercapnia on upper airway resistance and collapsibility in anesthetized dogs. Respir Physiol 75: 29-38, 1989.

29. Permutt S, Bromberger-Barnea B, and Bane HN. Alveolar pressure, pulmonary venous pressure, and the vascular waterfall. Med Thorac 19: 239-260, 1962.

30. Permutt S and Riley RL. Hemodynamics of collapsible vessels with tone: the vascular waterfall. J Appl Physiol 18: 924-932, 1963.

31. Philip-Joet F, Marc I, and Series F. Effects of genioglossal response to negative airway pressure on upper airway collapsibility during sleep. J Appl Physiol 80: 1466-1474, 1996.

32. Pride NB, Permutt S, Riley RL, and Bromberger-Barnea B. Determinants of maximal expiratory flow from the lungs. J Appl Physiol 23: 646-662, 1967.

33. Remmers JE, deGroot WJ, Sauerland EK, and Anch AM. Pathogenesis of upper airway occlusion during sleep. $J \mathrm{Appl}$ Physiol 44: 931-938, 1978.

34. Rowley JA, Permutt S, Willey S, Smith PL, and Schwartz AR. Effect of tracheal and tongue displacement on upper airway airflow dynamics. J Appl Physiol 80: 2171-2178, 1996.

35. Rowley JA, Williams BC, Smith PL, and Schwartz AR. Neuromuscular activity and upper airway collapsibility. Mechanisms of action in the decerebrate cat. Am J Respir Crit Care Med 156: 515-521, 1997.

36. Sauerland EK and Harper RM. The human tongue during sleep: electromyographic activity of the genioglossus muscle. Exp Neurol 51: 160-170, 1976.

37. Sauerland EK, Sauerland BA, Orr WC, and Hairston LE. Non-invasive electromyography of human genioglossal (tongue) activity. Electromyogr Clin Neurophysiol 21: 279-286, 1981.

38. Schneider H, O'Hearn DJ, Leblanc K, Smith PL, O'Donnell CP, Eisele DW, Peter JH, and Schwartz AR. High-flow transtracheal insufflation treats obstructive sleep apnea. A pilot study. Am J Respir Crit Care Med 161: 1869-1876, 2000.

39. Schwartz AR, Gold AR, Schubert N, Stryzak A, Wise RA, Permutt S, and Smith PL. Effect of weight loss on upper airway collapsibility in obstructive sleep apnea. Am Rev Respir Dis 144: 494-498, 1991.

40. Schwartz AR, O'Donnell CP, Baron J, Schubert N, Alam D, Samadi SD, and Smith PL. The hypotonic upper airway in obstructive sleep apnea: role of structures and neuromuscular activity. Am J Respir Crit Care Med 157: 1051-1057, 1998.

42. Schwartz AR, Schubert N, Rothman W, Godley F, Marsh B, Eisele D, Nadeau J, Permutt L, Gleadhill I, and Smith PL. Effect of uvulopalatopharyngoplasty on upper airway collapsibility in obstructive sleep apnea. Am Rev Respir Dis 145: 527-532, 1992.

43. Schwartz AR, Smith PL, Wise RA, Bankman I, and Permutt S. Effect of positive nasal pressure on upper airway pressure-flow relationships. J Appl Physiol 66: 1626-1634, 1989.

44. Schwartz AR, Smith PL, Wise RA, Gold AR, and Permutt S. Induction of upper airway occlusion in sleeping individuals with subatmospheric nasal pressure. J Appl Physiol 64: 535$542,1988$.

45. Schwartz AR, Thut DC, Brower RG, Gauda EB, Roach D, Permutt S, and Smith PL. Modulation of maximal inspiratory airflow by neuromuscular activity: effect of $\mathrm{CO}_{2}$. J Appl Physiol 74: 1597-1605, 1993. 
46. Seelagy MM, Schwartz AR, Russ DB, King ED, Wise RA, and Smith PL. Reflex modulation of airflow dynamics through the upper airway. J Appl Physiol 76: 2692-2700, 1994.

47. Shea SA, Akahoshi T, Edwards JK, and White DP. Influence of chemoreceptor stimuli on genioglossal response to negative pressure in humans. Am J Respir Crit Care Med 162: $559-565,2000$.

48. Skatrud JB, Dempsey JA, Badr S, and Begle RL. Effect of airway impedance on $\mathrm{CO}_{2}$ retention and respiratory muscle activity during NREM sleep. J Appl Physiol 65: 1676-1685, 1988.

49. Smith PL, Wise RA, Gold AR, Schwartz AR, and Permutt S. Upper airway pressure-flow relationships in obstructive sleep apnea. J Appl Physiol 64: 789-795, 1988.

50. Sullivan CE, Saunders NA, Issa FG, and Berthon-Jones M. Pathophysiology of sleep apnea. In: Sleep and Breathing, edited by Saunders NA and Sullivan CE. New York: Dekker, 1984, vol. 21, p. 299-363. (Lung Biol. Health Dis. Ser.)

51. Tantucci C, Duguet A, Ferretti A, Mehiri S, Arnulf I, Zelter M, Similowski T, Derenne JP, and Milic-Emili J. Effect of negative expiratory pressure on respiratory system flow resis- tance in awake snorers and nonsnorers. J Appl Physiol 87: 969-976, 1999.

52. Thut DC, Schwartz AR, Roach D, Wise RA, Permutt S, and Smith PL. Tracheal and neck position influence upper airway airflow dynamics by altering airway length. J Appl Physiol 75: 2084-2090, 1993.

53. Tuxen DV. Detrimental effects of positive end-expiratory pressure during controlled mechanical ventilation of patients with severe airflow obstruction. Am Rev Respir Dis 140: 5-9, 1989.

54. Van de Graaff WB. Thoracic influence on upper airway patency. J Appl Physiol 65: 2124-2131, 1988.

55. Van de Graaff WB. Thoracic traction on the trachea: mechanisms and magnitude. J Appl Physiol 70: 1328-1336, 1991.

56. Van Lunteren E, Strohl KP, Parker DM, Bruce EN, Van de Graaff WB, and Cherniack NS. Phasic volume-related feedback on upper airway muscle activity. J Appl Physiol 56: 730736, 1984.

57. West JB. Blood flow. In: Respiratory Physiology-The Essentials. Baltimore, MD: Williams \& Wilkins, 1975, chapt. 4, p. 33-50.

58. Wheatley JR, Mezzanotte WS, Tangel DJ, and White DP. Influence of sleep on genioglossus muscle activation by negative pressure in normal men. Am Rev Respir Dis 148: 597-605, 1993.

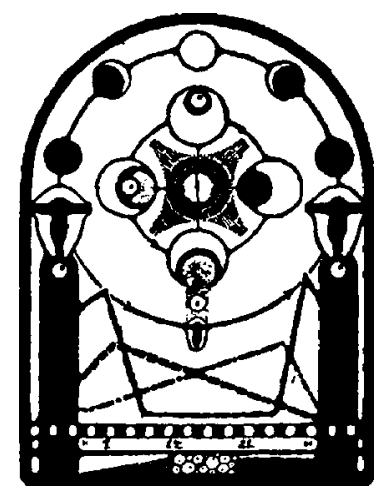

